\title{
RAPID AND FACILE FABRICATION OF 3D-NETWORKED CIRCULAR MICROFLUIDIC CHANNELS IN PDMS
}

\author{
Jiwon Lee, Jungwook Paek, and Jaeyoun Kim* \\ Department of Electrical and Computer Engineering, \\ Iowa State University, Ames, Iowa, USA
}

\begin{abstract}
In this paper, we propose a new fabrication method which establishes a simple, rapid, and cost-effective way of generating 3D-networked circular microfluidic (MF) channels. Due to the growing interests to mimic biological vasculatures [13], recently techniques to develop circular MF channels with 3D networks are on demand. By adapting sucrose fibers as eco-friendly sacrificial templates, we established rapidly prototype pre-designed scheme which does not require cumbersome fume-hoods or cleanroom facilities. Moreover, easy control of thicknesses and easy modification of the structure coming from the water-dissolvable nature of sucrose fiber makes various structures feasible in 3Dnetworked circular MF applications.
\end{abstract}

\section{INTRODUCTION}

2D-networked rectangular microfluidic (MF) channels have been the workhorse of lab-on-a-chip (LoC) devices. Recent expansion in the LoC's scope of application, however, introduced an increasingly diverse set of requirements on the MF channel's cross-sectional shape and topology. Of special interest among them include the demands for MF channels with circular crosssection [1-2] and their non-planar 3D deployment [3-4]. While these new architectures will certainly benefit the on-going efforts to mimic biological vasculatures, the fabrication schemes have been complicated and limited as well in their ability to realize MF channel junctions that are physiologically realistic [5].

Circular MF channels have been fabricated through poly (dimethylsiloxane) (PDMS) molding of cylindrical templates predominantly with a variety of templates. Templates with sufficient strength, such as metal wires and nylon threads were physically pulled out, but the scheme cannot produce internally starting and ending MF channels within the PDMS blocks [6]. The use of chemically etchable templates allowed more complex geometries but inevitably brought complicated chemical processes which in general are time-consuming and high-temperature processes. As an alternative, rectangular MF channels were circularized by filling them with curable liquid and applying a strong air stream which bores cylindrical openings. Such a scheme is advantageous for its applicability to conventionally prepared 2D MF networks, whereas it required additional steps involving special solvents.

The realization of non-planar MF networks to study biological systems has also been pursued actively. Layer-by-layer assembly of planar structures [6-7], with vertical via connecting MF channels in different layers, has been the most commonly adopted whereas it was not true 3D MF channels trajectories. Instead, in the plug-in-and-mold scheme, PDMS MF "pipes" were cut out from a 2D MF network prepared in advance and then manually shaped into 3D topologies before getting immersed in liquid-phase PDMS to be cured. Another more recent scheme, omni-directional printing, fabricated 3D MF channel networks by depositing filaments of fugitive ink that can be liquefied and drained after the embedding material got solidified [8].

Thus, a new fabrication scheme should accomplish both circular MF channels and 3D trajectories simultaneously. In particular, it is highly desired to establish a simple and accessible, yet flexible, rapid prototyping technique for 3D-networed circular MF channels. Some of the previously mentioned schemes actually meet the dual requirements. The omni-directionally printed 3D MF channels [8] exhibit circular cross-section thanks to the filamentary morphology of the nozzle-injected ink. The schemes in Refs [8], [9], and [10] also accomplished the dual goal using 3D-shapable cylindrical templates as well as complex instruments and materials. These schemes, however, are not suitable for readily accessible rapid prototyping or compatible with PDMS. Another attempt, the simple overlapping method of two cylindrical templates would result in a physiologically un-realistic junction shape with little contact area. The formation of a proper junction requires attaching one end of the template to the side of another. With the template materials not providing effective ways for the task, the authors of the previous work had to fuse the overlapping areas mechanically or thermally, a cumbersome process that may further deform the junction shape.

In this work, we demonstrate a new scheme for rapid prototyping of MF channels with circular cross-sections and 3D trajectories, with an emphasis on realizing physiologically realistic channel branches. Our work differs from the previous ones in providing a scheme to rapidly prototype pre-designed, rather than randomly shaped, 3D networks of circular MF channels. We also focus on achieving the dual goal using low-temperature, waterbased processes so that the need for fume-hoods or cleanroom facilities can be eliminated. In this regard, we established techniques to make junctions between the sucrose fiber templates and devised methods to efficiently control the trajectories of the templates. The technique is also compatible with a rapid prototyping method, the conventional PDMS molding process. In addition, we exploited the wide variety in the achievable shape of the sucrose template to implement structures other than MF channels as well.
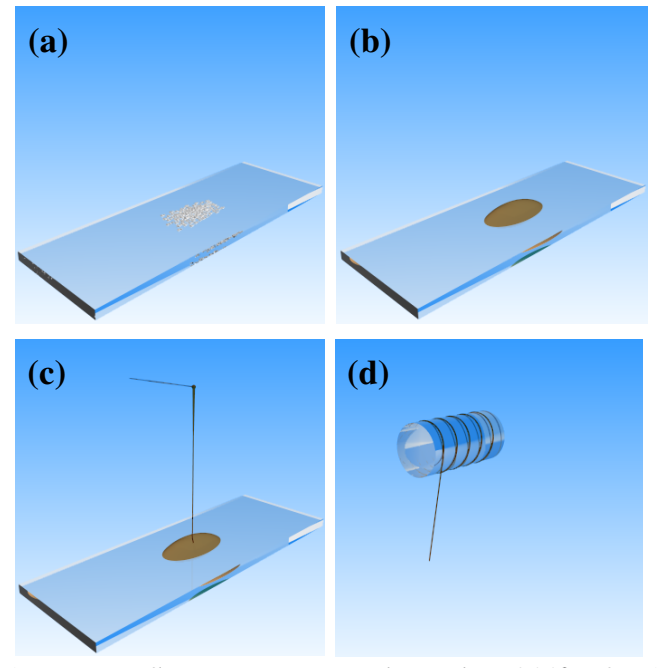

Figure 1: Process flow (a) sucrose is heated on $200^{\circ} \mathrm{C}$ for $15 \mathrm{~min}$, (b) at light brown color, ramp down to $125^{\circ} \mathrm{C}$ for 10min, (c) pull sucrose fibers at $0.1 \sim 0.5 \mathrm{~m} / \mathrm{s},(d)$ coil it around a cylindrical PDMS base template 


\section{MATERIAL SELECTION}

The key enabling factor is the use of sucrose as the sacrificial material. It is highly water-soluble and can be pulled into cylindrical fibers at $<200^{\circ} \mathrm{C}$ for various thicknesses. The resulting fibers also exhibit mechanical strength and surface smoothness adequate for handling/shaping and formation of transparent MF channels, respectively. These advantageous features of sugarbased sacrificial template materials have been exploited formerly. Bellan et al [11] fabricated 3D artificial vascular structures by melt-spinning random sugar fiber networks with a cotton-candy machine and then replicating them with PDMS, epoxy, and poly (caprolactone). Li et al [12] also fabricated bundles of poly-(Llactic acid) (PLLA) tubules for nerve scaffolds by coating sucrose fibers with PLLA and then dissolving the fibers. Former studies, however, are targeted random or simple tubular structures only. We fully exploit the mechanical and thermal properties of sucrose fiber to realize pre-designed templates in a more controlled fashion.

PDMS molding, which is popularly used for its easy curing mechanism, transparency, and flexibility, is selected as to generate MF structures. Due to the temperature budget and chemical-free dissolving mechanism of sucrose, it is suitable to be used with PDMS as well as it enables the fabrication of complex MF channels in bench-top settings. Figure 2 offers an example of the structure created with sucrose as a sacrificial 3D template for the PDMS based MF structure.
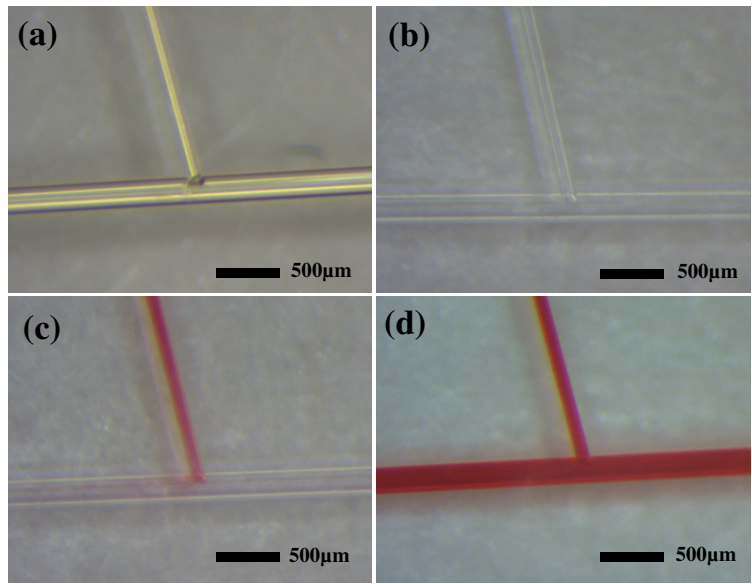

Figure 2: Microscopic images of a PDMS MF junction made with two sucrose fibers: (a) original sucrose fiber assembly, (b) after dissolving the sucrose, $(c-d)$ red food dye was injected to visualize the junction

\section{FABRICATION METHOD}

\section{Preparation of sucrose fibers}

Steps for sucrose fiber pulling are described in Figs. 1(a)-(c). First, crystalline sucrose was melted at $200^{\circ} \mathrm{C}$. Upon turning into light-brown color after 10-15 $\mathrm{min}$, the temperature was ramped down to $125^{\circ} \mathrm{C}$ and maintained for additional $10 \mathrm{~min}$. This particular caramelization temperature extends the duration of the caramelized state up to 3 hours. Then sucrose fibers were pulled at $0.1-0.5 \mathrm{~m} / \mathrm{s}$ with an optical fiber. The diameter of the pulled sucrose fiber was varied between few tens to several hundred micrometers rely on the speed. Both manual pulling and micromanipulator automated pulling is possible depending on the length and the thickness of fiber required.

\section{Assembly of sucrose fibers}

The water-soluble nature and high tensile strength of the sucrose fibers facilitates their manual handling and assembly. Sucrose fibers pulled out of caramelized sucrose instantly solidify. Subsequently, pulled fibers were assembled into a 3D network on or around the base PDMS template. First, the PDMS base plate was made with standard PDMS process. Sylgard184 base and curing agent were mixed at 10:1 ratio and cured for 3 hours at $60^{\circ} \mathrm{C}$. Then, two or more sucrose fibers are located on top of the base plate, used as substrate of final product. Subsequently, one end of a sucrose fiber was closely applied to the cylindrical body of another sucrose fiber to create junctions. Then, two sucrose fibers were easily attached to each other by applying small amount of water. Figure 2(a) shows original sucrose fibers made with above method. To secure the bonding, adhesives made with additional sucrose and water as $2: 3$ weight-ratios and heated on $90^{\circ} \mathrm{C}$ hot plate temperature could also be used. In addition, sucrose-water adhesives make different junction angles feasible, but large quantity of them can cause residues while water should be continuously supplied to maintain the optimum weight-ratio. Not only multiple branches were made by connecting sucrose fibers, but also with a lengthy single fiber we could make different types of patterns. In general, we can easily withdraw a couple meters of a thin sucrose fiber. Especially when the diameter of sucrose fiber is less than $50 \mu \mathrm{m}$, it is flexible enough to be coiled or located around any shapes depending on the PDMS template. For instance, a sucrose fiber can be coiled around a PDMS cylinder to make a solenoidal MF structure as illustrated in Fig. 1(d).

\section{Dissolving of Sucrose fibers}

After being assembled on or around the PDMS base template, sucrose template structure was placed inside a container and subsequently filled with another batch of PDMS. Once PDMS is completely cured, the sucrose fiber assembly was dissolved in warm $\left(\sim 70^{\circ} \mathrm{C}\right)$ water. This low temperature, water-based template removal makes our scheme much simpler than the other methods relying on chemical etching. To aid the complete removal of sucrose, flow of warm water was injected with a syringe.

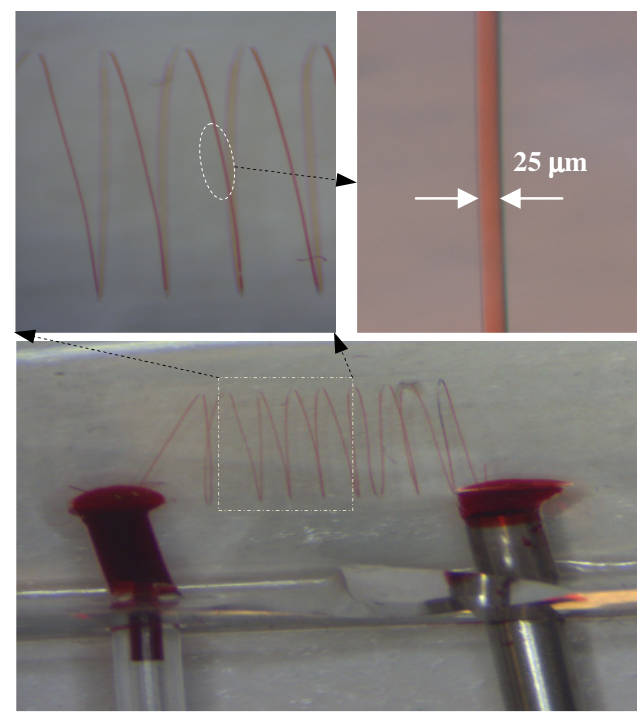

Figure 3: Microscopic images of a $25 \mu$ m-diameter MF channel forming a 3D solenoidal network with a $5 \mathrm{~mm}$-diameter size core 


\section{RESULT AND DISCUSSION}

Microfluidic channels

Figure 2 (a-d) shows a MF channel smoothly branching from a bigger one. From the initial sucrose fiber template (Fig. 2(a)) to the water-filled MF channel in PDMS (Fig. 2(b)), we can observe smooth surface. Surface quality and the circular shape of sucrose fiber are characterized further in the following sections. Especially, the pattern of gradual spreading red food dye (Fig. 2(c)) affirms that the junction was formed at the side of the bigger channel, a proper form for vaso-mimetic applications.

Not only simple junctions but we also created a MF channel with solenoidal trajectory as shown in Fig. 3. The diameter of the MF channel and the solenoid is $25 \mu \mathrm{m}$ and $5 \mathrm{~mm}$, respectively. Like the presented solenoidal structure, we can generate various structures with long yet compact vessel-like structures according to the base template trajectory and how we embed sucrose fibers.
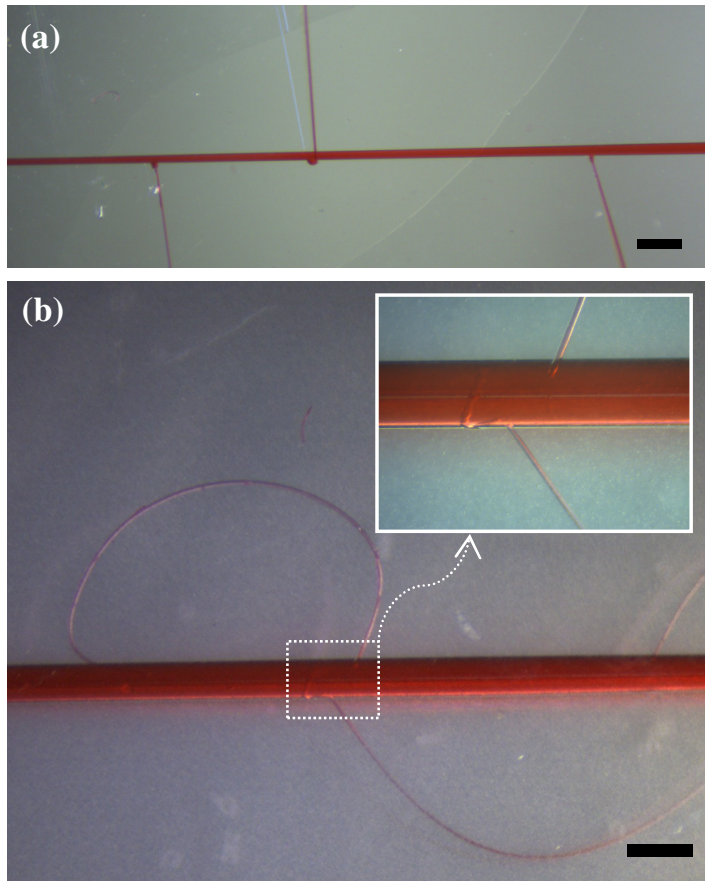

Figure 4: Microscopic images of PDMS MF structures filled with red dyes (a) branches made with assembly of straight fibers (b) $A$ $3 D$ assembly of curved fibers attached at different angles which start and end internally. And the inlet shows magnified image of its junctions. Scale bars: $1 \mathrm{~mm}$

\section{D networked trajectories}

Various structures can be realized due to the waterdissolvable nature of sucrose as introduced in the former fabrication sections. Especially the power of this technique reveals when we make angled 3D structures. Not only angles in the azimuth direction but also in the elevation direction are feasible by simply applying of trapezoidal pyramid PDMS block template with different angles underneath each sucrose fiber. When higher quantity of water-sucrose adhesives is applied, it is also possible to make the same angled branch structure in both directions without having supporting blocks. For example, the structure shown in the Fig 4(a) provides the three junctions made only with adhesive strength. Due to the tapering, the thickness of the center channel varies that the average thickness is $220 \mu \mathrm{m}$. The thicknesses of branches fall into the range of $60 \pm 5 \mu \mathrm{m}$.
Figure 4(b) illustrates internally looped MF channels embedded in the PDMS molding. There are three internally starting and ending fibers in the figure, but two of them are cropped to enhance the visibility. All three curved structures are shaped by applying steam prior to attachment. Also, the inlet of Fig. 4(b) shows magnified image of junctions to illustrate their smooth and angled transition of the structure. The thickness of the center channel is $586 \mu \mathrm{m}$ while branches are measured as $53 \mu \mathrm{m}$ for the top one and $45 \mu \mathrm{m}$ for the bottom one accordingly. Particularly, this structure demonstrates the great potentials in obtaining various shapes and size structures. The location and the angle of junctions can be further controlled by micro-manipulator. Especially, the images prove its physiologically realistic bifurcation of MF channels.

\section{D shaped and tapered structure}

Figure 5(a) illustrates a tapered and 3D shaped MF channel. By starting with the thicker pulling rod and varying the instant pulling speed, we could manually make a tapered structure. Then, a point of the pulled sucrose fiber was exposed to steam for a few seconds while forming a curve. Instant removal of steam is required to prevent breaking of the fiber. This effortless tapering and shaping offer great potentials of generating more complicated 3D networks. In particular, with the right equipment set-up, such as a computer-controlled manipulator, more complicated and compact drawing to realize vaso-mimetic structures are possible.

Figure 5(b-c) shows microscopic images of an original sucrose fiber. This particular sucrose fiber is obtained by manually withdrawn for $51 \mathrm{~cm}$ and timed as $3.4 \mathrm{sec}$. Then, to avoid the abrupt change of thickness, only the mid-point of the fiber is used to be measured and averaged. The diameter of fiber is measured as $123 \mu \mathrm{m}$. As we observe in images, the both cylindrical body and the cut edge surfaces are smooth at the micron scale. Also, cross sectional view in (c) proves the circular shape of MF channel to contribute to the realization of the vaso-mimetic LoCs.

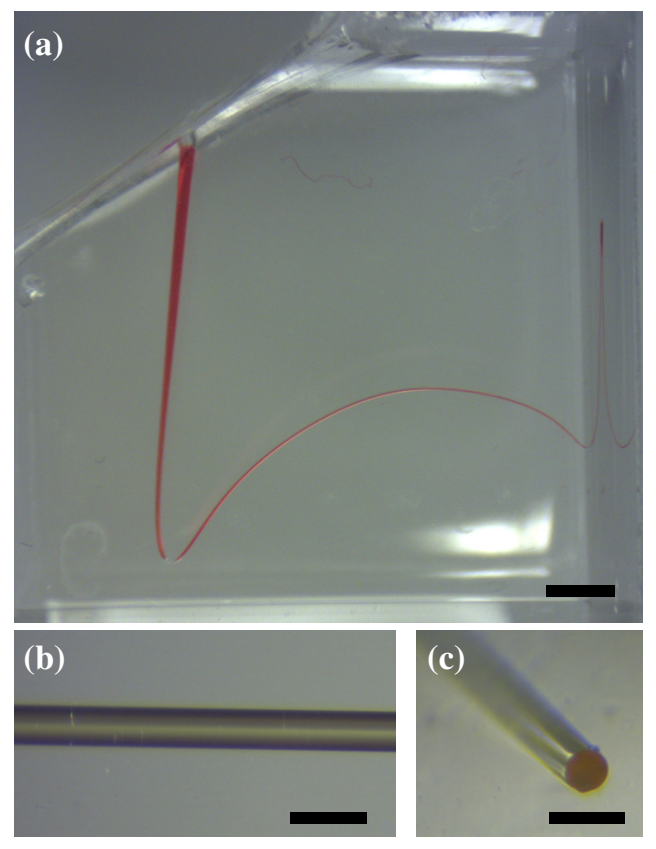

Figure 5: Microscopic images: (a) 3D MF channel made with a tapered sucrose fiber and shaped with steam $(b)$ the top view and (c) the angled-cross sectional view of the original sucrose fiber pulled at $3.4 \mathrm{~cm} / \mathrm{s}$. Scale bars: $250 \mu \mathrm{m}$ 

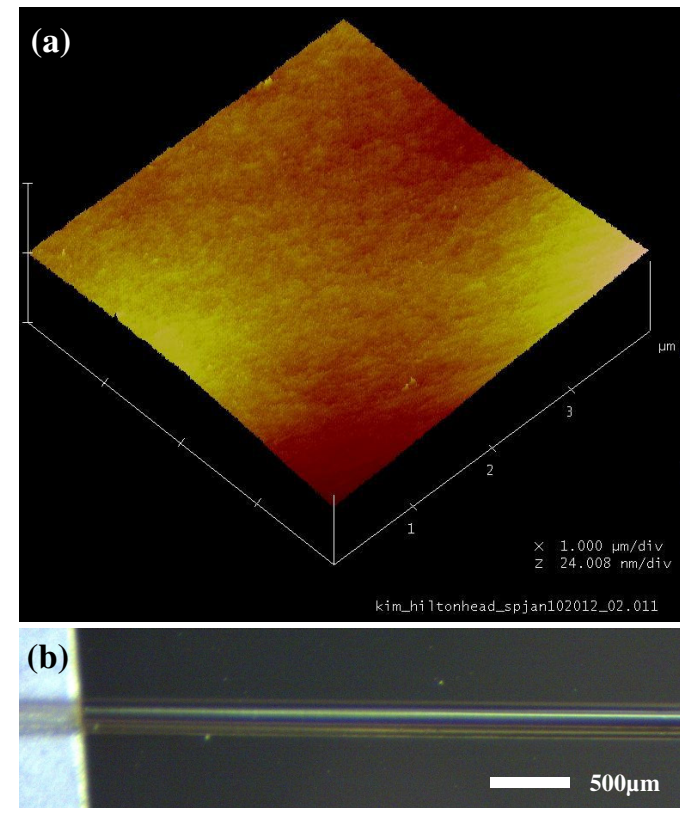

Figure 6: (a) 3D AFM scan of $16 \mu^{2}$ area of the outer surface of sucrose fiber, (b) a microscopic image of its original fiber

We further investigated surface roughness of the sucrose fiber by using AFM (atomic force microscopy) scan. Figure 6 shows the 3D AFM scans of $16 \mu \mathrm{m}^{2}$ area. The measurement scan was carried out over ten randomly selected locations on a single fiber. Then, Nanoscope program is used to characterize the surface. The root mean square roughness value was found to be $1.1 \pm 0.4 \mathrm{~nm}$, confirming its excellent surface smoothness.

\section{CONCLUSION}

In summary, we established a simple, rapid, and cost-effective fabrication scheme to realize 3D networks of circular MF channels by adopting sucrose as the sacrificial material. Various MF structures shown in the manuscript prove the great potential of our fabrication method. The excellent chemical, mechanical, and thermal properties of sucrose allowed a high level of flexibility in the attainable network topologies. Especially, the easy tapering and curving modification of the sucrose template can be greatly used for the formation of physiologically realistic junctions.

\section{ACKNOWLEDGEMENT}

The authors were supported by NSF CAREER award (0954845).

\section{REFERENCES}

[1] M. Abdelgawad, C. Wu, W.-Y. Chien, W. R. Geddie, M. A. S. Jewett and Y. Sun, "A fast and simple method to fabricate circular microchannels in polydimethylsiloxane (PDMS)," Lab on a Chip, 11, 545 (2011).

[2] S.-H. Song, C.-K. Lee, T.-J. Kim, I.-C. Shin, S.-C. Jun and H.-I. Jung, "A rapid and simple fabrication method for 3dimensional circular microfluidic channel using metal wire removal process," Microfluid Nanofluid, 9, 533 (2010).

[3] H. Wu, T. W. Odom, D. T. Chiu, and G. M. Whitesides, "Fabrication of Complex Three-Dimensional Microchannel Systems in PDMS," J. Am. Chem. Soc., 125, 554-559 (2012).

[4] Y. Luo and R. N. Zare, "Perforated membrane method for fabricating three-dimensional polydimethylsiloxane microfluidic devices," Lab Chip, 8, 1688 (2008).

[5] J. M. Rosano, N. Tousi, R. C. Scott, B. Krynska, V. Rizzo, B. Prabhakarpandian, K. Pant, S. Sundaram, and M. F. Kiani, "A physiologically realistic in vitro model of microvascular networks," Biomed Microdevices, 11, 5 (2009).

[6] M. K. S. Verma, A. Majumder, and A. Ghatak, "Embedded Template-Assisted Fabrication of Complex Microchannels in PDMS and Design of a Microfluidic Adhesive," Langmuir, 22, 10291-10295 (2006).

[7] Y. Jia, J. Jiang, X. Ma, Y. Li, H. Huang, K. Cai, S. Cai, and $\mathrm{Y}$. Wu, "PDMS microchannel fabrication technique based on microwire-molding," Chinese Science Bulletin, 53, 39283936 (2008).

[8] W. Wu, A. DeConinck, and J. A. Lewis, "Omnidirectional Printing of 3D Microvascular Networks," Advanced Materials, 23, H178-H183 (2011).

[9] Y. Du, M. Ghodousi, H. Qi, N. Haas, W. Xiao, and A. Khademhosseini, "Sequential assembly of cell-laden hydrogel constructs to engineer vascular-like microchannels," Biotechnology and Bioengineering, 108, 1693-1703 (2011).

[10] C. Xia and N. X. Fang, "3D microfabricated bioreactor with capillaries," Biomedical Microdevices 11, 1309-1315 (2009).

[11] L. M. Bellan, S. P. Singh, P. W. Henderson, T. J. Porri, H. G. Graighead and J. A. Spector, "Fabrication of an artificial 3dimensional vascular network using sacrificial sugar structures," Soft Matter, 5, 1354 (2009)

[12] J. Li, T. A. Rickett, and R. Shi, "Biomimetic Nerve Scaffolds with aligned Intraluminal Microchannels: A "Sweet" Approach to Tissue Engineering," Langmuir, 25, 1813 (2009)

[13] L. K. Fiddes, N. Raz, S. Srigunapalan, E. Tumarkan, C. A. Simmons, A. R. Wheeler, E. Kumacheva, "A circular crosssection PDMS microfluidics system for replication of cardiovascular flow conditions," Biomaterials, 31, 3459 (2010).

\section{CONTACT}

*Jaeyoun Kim, tel: +1-515-294-4214; plasmon@istate.edu 